\author{
Marija Vučinić ${ }^{*}$
}

\title{
L'UNITÀ DEL PREDICATO NEI COSTRUTTI INFINITIVI RETTI DAI VERBI MODALI
}

\begin{abstract}
In questo nostro contributo intendiamo offrire una descrizione sintattica dei costrutti infinitivi retti dai verbi modali, concentrandoci in particolare sulla questione dell'unità del sintagma verbale. In questa ricerca abbiamo analizzato le proprietà semantiche dei verbi modali e i seguenti fattori sintattici come tratti distintivi che potrebbero incidere sulla coerenza del costrutto e differenziare la costruzione monofrasale dalla costruzione bifrasale: la posizione dei pronomi atoni, la scelta dell'ausiliare, l'uso dell'infinito composto, la posizione della negazione, l'aspetto verbale. Il corpus preso in esame è diacronico e comprende le opere letterarie degli autori che hanno segnato in maniera determinante lo sviluppo della sintassi e della prosa volgare italiana. Abbiamo condotto un'analisi qualitativa, tralasciando l'aspetto statistico, che pur essendo di grande interesse, manca nei corpora da noi esaminati. Il presente lavoro si propone di dare una descrizione sintattica dei costrutti infinitivi retti dai verbi modali nell'italiano antico, così che si possono ricavare indicazioni utili per descriverne la struttura e avanzare alcune possibili interpretazioni dell'unità del sintagma verbale.

Parole chiave: infinito, verbi modali, costruzione monofrasale, costruzione bifrasale, unità del predicato, italiano antico.
\end{abstract}

\section{INTRODUZIONE}

L'infinito subordinato direttamente al verbo apre la complessa questione dell'unità del sintagma verbale.

L'analisi prende in esame costrutti infinitivi retti dai verbi modali che possono formare un unico complesso verbale insieme all'infinito (costruzione monofrasale), oppure costituire due predicati separati (costruzione bifrasale). Ci sono varie interpretazioni linguistiche riguardo all'unità sintattica dei costrutti infinitivi retti dai verbi modali e alla funzione dei loro membri sul piano sintattico e semantico. Nel presente lavoro saranno

*vucinicmarija@yahoo.com 
analizzati diversi punti di vista e delle ipotesi che si discostano dall'impostazione della grammatica tradizionale, secondo la quale i verbi modali sono considerati verbi servili ${ }^{1}$.

Verranno esaminati i valori semantici dei verbi modali, i fattori sintattici che possano incidere sulla coerenza del costrutto, e verranno confrontate infine le loro proprietà sintattiche e semantiche che differenziano la costruzione monofrasale da quella bifrasale.

Il corpus che abbiamo preso in esame è di tipo diacronico e comprende le opere letterarie risalenti a un periodo di tempo che va dal Duecento, quando il volgare si afferma pienamente nell'uso letterario, fino al tardo Rinascimento. Il corpus contiene le opere degli autori che hanno segnato in maniera determinante lo sviluppo della prosa volgare italiana e della letteratura italiana in genere. La scelta del corpus è stata dettata dal desiderio di dare una visione più completa sull'evoluzione sintattica della lingua italiana.

Considerate le dimensioni del corpus e la diversità dei testi esaminati (dalle opere dantesche scritte in lingua volgare, Vita Nuova, continuazione della tradizione letteraria medievale, e Il Convivio, il primo modello della prosa filosofica in volgare, fortemente influenzato dalla sintassi latina, attraverso un'opera giovanile di Giovanni Boccaccio, strutturata come un lungo monologo-confessione, e il Decameron, caratterizzato da un alto livello di complessità sintattica, fino ai trattati storico-politici di Machiavelli, Il Principe e Dell'arte della guerra, con dei modelli sintattici che si allontanano dalla tradizione umanistica latina), lo giudichiamo "un campione" abbastanza generico e rappresentativo della sintassi dell'italiano antico.

\section{LA SEMANTICA DEI VERBI MODALI}

Nei costrutti modali, a differenza degli altri costrutti nei quali l'infinito è collegato direttamente al verbo reggente (accusativo con l'infinito, costrutti causativi e percettivi), il soggetto dell'infinito coincide con quello del verbo modale:

[...] ha posto tutta la mia beatitudine in quello che non mi puote venire meno. (Vita nuova, XVIII, 244)

Sulla delimitazione del gruppo e sul numero dei verbi modali non c'è pieno accordo, ma nel presente lavoro si prendono in esame i costrutti modali con i verbi dovere, potere, volere, mentre sapere e solere, avendo il loro uso in funzione modale piuttosto ristretto rispetto agli altri verbi modali, saranno considerati verbi modali marginali ${ }^{2}$.

\footnotetext{
${ }^{1}$ Cfr. Dardano \& Trifone (1997); Serianni (1989); Simone \& Amacker (1977).

${ }^{2}$ Cfr. Skytte (1983); Skytte \& Salvi (1991).
} 
L'analisi della modalità sembra essere oggetto di un robusto interesse linguistico. Nello studio della modalità dell'enunciato, concepita come grammaticalizzazione dell'atteggiamento soggettivo del parlante, un ruolo centrale viene attribuito al modo verbale ${ }^{3}$.

Secondo Simone \& Amacker (1977), i verbi modali in italiano non hanno peculiarità sintattiche che permettano di individuare una classe di modali distinta da altri verbi. I verbi modali non sono i soli verbi italiani che precedono immediatamente il verbo all'infinito, né i soli a permettere la relativa libertà riguardo alla posizione dei clitici ${ }^{4}$.

Nella nostra analisi, la struttura sintattica dei costrutti modali, e più precisamente la questione dell'unità del predicato, sarà esaminata e descritta a partire dai tratti semantici, i quali insieme ai fattori sintattici possono incidere sulla coerenza del costrutto e sull'opposizione unità / dualità 5 .

Nell'esaminare la struttura sintattica delle costruzioni modali, sarebbe opportuno partire da un'approfondita analisi dei loro tratti semantici, visto che la modalità rappresenta l'atteggiamento del parlante verso il contenuto. Si ritiene utile riprendere la riflessione di Pietrandrea (2005: 6) sul carattere universale della modalità, considerata uno dei tratti fondamentali di ogni atto illocutorio, che mette il parlante "in relazione con la sua enunciazione". La modalità può essere sottintesa e recuperata attraverso il contesto situazionale ed è spesso relativa all'opinione del parlante. Quest'attenzione nei confronti degli aspetti semantici viene spiegata con il fatto che i verbi possono essere considerati modali grazie appunto ai tratti semantici che li contraddistinguono.

Si distinguono due tipi principali di modalità: modalità epistemica e modalità deontica (Bybee et al. 1994; Lyons 1977; Palmer 1990).

Nel senso epistemico, i verbi modali rimandano alle nozioni di probabilità (dovere) o possibilità (potere) $)^{6}$ :

[...] e lasciate fare a me, ché fermamente io acconcerò i fatti vostri ed i miei in maniera che starà bene $\mathrm{e}$ che dovrete esser contenti. (Decameron, $\mathrm{I}, 1,32$ )

${ }^{3}$ Cfr. Palmer (1986, 2003).

${ }^{4}$ Cfr. Skytte (1983: 78): "Il costrutto che, forse, somiglia di più al costrutto di verbo modale + inf. è continuare, cominciare + a Inf; nonché stare, andare, venire + a Inf: ha cominciato ad alzarsi, si è cominciato ad alzare, lo vado a invitare, vado a invitarlo".

${ }^{5}$ Cfr. Skytte (1983: 41): "Che due verbi si uniscano in un solo sintagma, vuol dire che si comportano come un verbo singolo sul livello della proposizione, cioè esprimono insieme il tempo, il modo, la diatesi e per quanto riguarda la valenza e la semantica, si comportano come un'unità sul livello della proposizione".

${ }^{6}$ Cfr. Serianni (1989). 
Chi dee mai essere lieta di noi, che avemo udita parlare questa donna così pietosamente? (Vita nuova, XXIII, 250)

Che essi non sien tutti veri, assai volte può ciascun di noi aver conosciuto... (Decameron, IV, 6, 308)

E secondo malizia, ovvero difetto di corpo, può essere la mente non sana... (Convivio, IV, XV, 340)

I costrutti modali nel senso epistemico hanno spesso un significato impersonale:

E non puote essere che con quella pietosa donna non sia nobilissimo amore. (Vita nuova, XXXV, 257)

E puote essere che il cielo in quella parte è più spesso... (Convivio, II, XV, 294)

Il senso epistemico dei costrutti modali è attestato già nel latino classico. Uno dei primi esempi del dovere (debere) annota Fleischman (1982, secondo Pietrandrea 2005: 204):

Sex pondo et seliberam debet habere. (Petronius, Satyricon, LXVII)

L'uso epistemico dei costrutti modali, specifica Pietrandrea (2005), fa emergere la soggettività del parlante e il legame che si crea fra il parlante e il suo enunciato. Analizzando la categoria della modalità, Halliday (1970) definisce propriamente modale solo la modalità epistemica che deriva dalla funzione interpersonale, espressiva e sociale del linguaggio e presuppone la partecipazione attiva del parlante.

A differenza della modalità epistemica che mette in rilievo il fattore semantico e l'atteggiamento del parlante verso l'enunciato, la modalità deontica riflette il carattere pragmatico del linguaggio: ci riferiamo a quei costrutti modali che esprimono una condizione di obbligo, permesso, necessità o possibilità, riferita al soggetto del costrutto, ma non voluta dal parlante ${ }^{7}$.

Nel senso deontico dovere esprime obbligo e potere permesso ${ }^{8}$ :

Se Francia, adunque, posseva con le forze sua assaltare Napoli, doveva farlo; se non poteva, non doveva dividerlo. (Principe, III, 9)

[...] vi si poteva per tutto andare. (Decameron, III, 184)

[...] debbe il principe leggere le istorie, e in quelle considerare le azioni degli uomini eccellenti; vedere come si sono governati nelle guerre... (Principe, XIV, 30)

\footnotetext{
${ }^{7}$ Cfr. Lyons (1977).

${ }^{8}$ Cfr. Serianni (1989); Skytte (1983).
} 
Mentre nel senso deontico i verbi dovere e potere esprimono un'azione che dipende dalla volontà, dal desiderio o dal bisogno di un soggetto esteriore, diverso da quello del verbo modale, nei costrutti modali con i verbi volere e sapere i due soggetti coincidono sul piano sintattico-semantico:

$[\ldots]$ con ciò fosse cosa che tutte le donne carolar sapessero [...] comandò la reina che gli strumenti venissero... (Decameron, I, 25)

[...] ho meco stesso proposto di volere, in quel poco che per me si può, in cambio di ciò che io ricevetti, ora che libero dirmi posso, e se non a coloro che me aiutarono, alli quali per avventura per lo lor senno o per la loro buona ventura non abbisogna, a quegli almeno a' quali fa luogo alcuno alleggiamento prestare. (Decameron, Proemio, 4)

Nei costrutti passivi il contesto serve a precisare il significato del verbo modale e assicurarne la giusta interpretazione. Nei costrutti del genere il verbo modale è prevalentemente usato in senso epistemico:

Qualunque affari, qualunque altre cagioni costà trovasti, già deono essere finite... (Elegia di Madonna Fiammetta, V, 37)

[...] per avventura gli potrebbe venir fatto quel che egli disiderava. (Decameron, VII, 7, 482)

Similmente, l'infinito di forma composta con valore temporale è tipico del senso epistemico'

Carissimi giovani, la nostra usanza vi può aver renduti certi quanto sia l'amore che io vi porto... (Decameron, IV, 3, 294)

Donne mie care, voi potete, così come io, molte volte avere udito che a niuna persona fa ingiuria chi onestamente usa la sua ragione. (Decameron, I, 18)

[...] fermamente avarizia non mi dée avere assalito per uomo di picciolo affare; qualche gran fatto dee essere costui che ribaldo mi pare... (Decameron, I, 7, 61)

L'autonomia temporale dell'infinito nei costrutti modali con l'infinito composto viene considerata da Skytte (1983: 90) uno dei fattori "che normalmente creano la dualità". Il termine epistemico, precisa Lyons (1977), indica la struttura logica di un enunciato che esprime credenze, atteggiamenti, possibilità, probabilità. In un tale contesto è accettabile l'interpretazione bifrasale del costrutto modale, visto che la modalità epistemica è intesa come atteggiamento del parlante nei confronti della proposizione, mentre la modalità deontica descrive semplicemente lo stato delle cose.

\footnotetext{
${ }^{9}$ Cfr. Schmitt Jensen (1973).
} 
Tuttavia, l'accentuata soggettività del parlante e il forte legame che si crea tra il parlante e il suo enunciato contribuiscono alla coerenza semantica del costrutto. Anzi, il significato primario dei verbi modali è leggermente modificato, ovvero attenuato, nella modalità epistemica, e perciò nei costrutti del genere, i verbi modali possono essere considerati verbi ausiliari ${ }^{10}$.

\section{I FATTORI SINTATTICI CHE POSSONO INCIDERE SULL'UNITÀ DEL COSTRUTTO}

Sulla coerenza dei costrutti infinitivi retti dai verbi modali esistono varie interpretazioni linguistiche. I verbi latini volo, nolo, possum, debeo, soleo, sono considerati verbi servili che richiedono un altro verbo di modo infinito del quale indicano una particolare modalità ${ }^{11}$.

I costrutti dovere e potere + infinito, precisa Rohlfs (1969), meritano una particolare attenzione. Il verbo dovere è considerato da Rohlfs (1969: 83) un verbo servile, per lo "strettissimo nesso sintattico tra il verbo servile e verbo principale, che fa sì che il rapporto temporale venga trasferito al primo verbo".

La coerenza del costrutto dovere + infinito si spiega con il fatto che la stessa azione può essere espressa in due modi:

- con l'infinito composto, retto da un verbo modale al presente: Deve aver osservato.

- con il verbo modale al passato: Ha dovuto osservare.

Tuttavia, l'infinito retto da un verbo modale viene spesso considerato complemento oggetto del verbo reggente potere, volere, dovere, sapere e solere (Ageno 1978; Egerland 2010; Skydsgaard 1977; Skytte 1983).

Nel presente lavoro saranno analizzati i seguenti fattori sintattici come tratti distintivi che possano incidere sulla coerenza del costrutto:

1. la posizione dei pronomi atoni;

2. la scelta dell'ausiliare;

3. l'uso dell'infinito composto;

4. la posizione della negazione;

5. l'aspetto verbale ${ }^{12}$.

${ }^{10}$ Cfr. Wurmbrand (1994).

${ }^{11}$ Cfr. Dardano \& Trifone (1997); Fornaciari (1881); Serianni (1989); Simone \& Amacker (1977).

${ }^{12}$ Cfr. Skytte (1983). 


\subsection{La posizione dei pronomi atoni}

La posizione dei pronomi atoni secondo molti linguisti potrebbe essere considerata un tratto distintivo dell'unità verbale. Partendo dal lavoro dei generativisti, concentrato sulla possibilità di realizzazione dei diversi tipi di costrutto, Lo Cascio (1970: 158) crede che lo spostamento del pronome atono (sostituente d'ordine) sia "facoltativo e non comporta alcuna modifica al contenuto del messaggio". Skytte (1983: 50) invece afferma che la possibilità di spostamento del pronome atono "non è facoltativa in tutti i casi" e descrive la differenza tra i due costrutti sul piano semantico: Lo posso vedere. Posso vederlo. Secondo l'interpretazione fatta da Skytte (ibid.), solo il primo costrutto (Lo posso vedere) può essere considerato "un sintagma verbale complesso". Secondo l'ipotesi di Lo Cascio, i due costrutti sarebbero identici sul piano sintattico-funzionale.

I verbi modali ammettono la ristrutturazione, il processo attraverso cui un verbo reggente può formare un unico complesso verbale insieme all'infinito $^{13}$. Nella forma ristrutturata i pronomi clitici si uniscono al verbo reggente. Nella forma non ristrutturata, i pronomi atoni si uniscono all'infinito e una tale costruzione viene interpretata come bifrasale (con due predicati separati):

Oh sventurati e malnati, che innanzi volete partirvi d'esta vita sotto il titolo d'Ortensio, che di Catone! (Convivio, IV, XXVIII, 357)

[...] niuna altra donna mai mi ti potrà tòrre. (Elegia di Madonna Fiammetta, V, 37)

L'interpretazione della struttura dei costrutti infinitivi retti dai verbi modali a partire dalla posizione dei pronomi atoni è sostanzialmente basata sulla loro struttura superficiale. La posizione dei pronomi atoni, cosiddetta salita dei clitici, non comporta invece nessuna modifica sul piano semantico. I verbi modali, semanticamente e sintatticamente, fungono da ausiliari che conferiscono all'azione espressa una specifica modalità e sono portatori di una funzione grammaticale ${ }^{14}$. Nel presente lavoro le costruzioni infinitive rette dai verbi modali saranno interpretate come un unico predicato complesso, indipendentemente dalla posizione dei clitici. La seguente tabella illustra la relativa libertà riguardo alla posizione dei clitici nelle costruzioni modali:

${ }^{13}$ Cfr. Egerland (2010: 830); Rizzi (1976: 8); Skytte \& Salvi (1991: 485).

14 Cfr. Wurmbrand (1994: 239): “modals are (restricted) quantifiers rather than lexical predicates". 
Tabella 1. La posizione dei clitici nelle costruzioni modali

\begin{tabular}{|c|c|c|}
\hline Il pronome & \multicolumn{2}{|c|}{ La posizione } \\
\hline & Unito al verbo modale & Unito all'infinito \\
\hline $\begin{array}{l}\text { Il pronome } \\
\text { atono } \\
\text { (accusativo / } \\
\text { dativo) }\end{array}$ & $\begin{array}{l}{[\ldots] \text { e volevala costringere di }} \\
\text { pigliar l'uno... (Decameron, } \\
\text { V, } 7,385)^{15} \\
{[\ldots] \text { quello che non mi puote }} \\
\text { venire meno. (Vita nuova, } \\
\text { XVIII, 244) } \\
\\
{[\ldots] \text { se io le vi volessi tutte }} \\
\text { contare.... (Decameron, VI, } \\
10,440)^{16}\end{array}$ & $\begin{array}{l}\text { Se Francia, adunque, posseva con } \\
\text { le forze sua assaltare Napoli, dove- } \\
\text { va farlo; se non poteva, non doveva } \\
\text { dividerlo. (Principe, III, 9) } \\
\text { [...] cosa alcuna che dispiacerci } \\
\text { debba... (Decameron, } \mathrm{I}, 1,31)\end{array}$ \\
\hline si riflessivo & $\begin{array}{l}{[\ldots] \text { se lo potevano godere }} \\
\text { oziosi... (Principe, IV, 11) } \\
\text { E puosselo el principe } \\
\text { guadagnare in molti modi... } \\
\text { (Principe, IX, 21) }\end{array}$ & $\begin{array}{l}{[\ldots] \text { là ov'io potea lamentarmi }} \\
\text { sanza essere udito. (Vita nuova, } \\
\text { XII, 240) } \\
\text { [...] come coloro li quali non si } \\
\text { possono muovere. (Vita nuova, } \\
\text { XXIII, 248) }\end{array}$ \\
\hline $\begin{array}{l}\text { Le particelle } \\
c i(v i) \text { e } n e\end{array}$ & $\begin{array}{l}\text { Udistù, in quella, cosa niuna } \\
\text { della quale tu dubiti o vogli- } \\
\text { ne domandare? (Decame- } \\
\text { ron, I, 6, 56) } \\
\text { Se egli sapesse lavorare } \\
\text { l'orto e volesseci rimanere... } \\
\text { (Decameron, III, 1, 188) }\end{array}$ & $\begin{array}{l}{[\ldots] \text { affermando che esservi doveva }} \\
\text { l'anello... (Decameron, II, } 5,112 \text { ) }\end{array}$ \\
\hline
\end{tabular}

${ }^{15}$ Si noti la posizione enclitica del pronome atono rispetto al verbo modale.

${ }^{16}$ L'ordine dei clitici nei cumuli non sempre corrisponde a quello dell'italiano moderno. Nel caso di due pronomi clitici l'accusativo precede il dativo, sia in proclisi che in enclisi: [...] questa donna meritamente è mia, né alcuno con giusto titolo la mi può raddomandare (Decameron, X, 4, 671); [...] niuna altra persona conosco che farlemi possa avere... (Decameron, III, 9, 253). 


\begin{tabular}{|c|c|}
\hline & Unito al verbo modale \\
\hline si impersonale & $\begin{array}{l}\text { [...] perché parlare non si può d'alcuno, che il parlatore non lodi o } \\
\text { non biasimi quelli, di cui egli parla. (Convivio, I, II, 266) } \\
{[\ldots] \text { dinanzi a la quale poco si potrebbe leggere... }} \\
\text { (Vita nuova, II, 235) }\end{array}$ \\
\hline si passivante & $\begin{array}{l}{[\ldots] \text { ma se ne sarieno assai potute annoverare di quelle che... }} \\
\text { (Decameron, I, 15) } \\
{[\ldots] \text { si ha a notare che gli uomini si debbano o vezzeggiare }} \\
\text { i spegnere... (Principe, III, 7) }\end{array}$ \\
\hline
\end{tabular}

Fra i tratti che si discostano dalla norma dell'italiano moderno, si possono individuare: la posizione enclitica dei pronomi atoni uniti al verbo modale e l'ordine dei clitici nei cumuli che non sempre corrisponde a quello dell'italiano moderno. A differenza di si riflessivo, che può essere unito sia al verbo modale che al verbo all'infinito, si impersonale e si passivante come in genere in italiano moderno, sono quasi sempre adiacenti al verbo reggente, il che contribuisce alla coerenza prosodica del complesso.

Nelle rare costruzioni con i due verbi modali, il pronome atono si unisce al verbo modale ${ }^{17}$. A differenza dell'italiano moderno, in tutti gli esempi individuati nel corpus, il pronome atono si unisce al primo verbo modale, sia in proclisi che in enclisi ${ }^{18}$ :

E se così fu, che so che fu, qual cagion vi dovea poter muovere a torglivi così rigidamente? (Decameron, III, 7, 229)

Questa non è troppo grave cosa, né troppo lunga, e deesi assai ben poter fare... (Decameron, III, 4, 209)

Nelle costruzioni modali con due infiniti coordinati che troviamo nelle subordinate implicite, il pronome atono può essere unito sia al verbo modale che al verbo all'infinito:

... non par doversi né potersi predicare... (Convivio, III, II, 299)

${ }^{17}$ Nella prosa quattrocentesca frequente è il caso di due verbi modali giustapposti, che Dardano (1963: 93) spiega con l'intento di "riprodurre cadenze latine": Vorrebbesi potere mantenere gli uomini immortali! (Libri della Famiglia, II, 53); [...] siano troppo obligati e suggetti a non potere né sapere volere o seguire se non quanto stimino essere accetto e grato a chi egli amino (Libri della famiglia, II, 40).

${ }^{18}$ Con i verbi modali al posto dell'infinito, in italiano moderno, il pronome atono si unisce al primo infinito, ovvero al secondo verbo modale (devi poterlo risolvere, Lo Cascio, 1970: 164). Secondo Lo Cascio (ibid.), questa struttura è la più frequente, anche se sono possibili altre due posizioni (Devi poter risolverlo. Lo devi poter risolvere, ibid.). 
Per tanto uno principe debbe esistimare poco [...] per potere defendersi... (Principe, XVI, 32)

[...] del tutto era disposto a volerlo fare impiccar per la gola ed in niuna guisa rendere il voleva al signore... (Decameron, II, 1, 82)

[...] e tanto affaticati erano che non poteano disfogare la mia trestizia, pensai di volere disfogarla con alquante parole dolorose... (Vita nuova, XXXI, 254)

Secondo Lo Cascio (1970: 159), la posizione del pronome atono "può essere dettata da esigenze stilistiche" e dal tipo di testo: la prosa scientifica preferirebbe le strutture in cui il pronome atono si unisca all'infinito, visto che nella maggioranza dei casi si tratta dell'uso epistemico, mentre nella lingua parlata e nel linguaggio poetico si trovano tutte e due le strutture. Le tendenze che dettano la scelta della posizione del pronome atono, spiega Skytte (1983), dipendono anche dai singoli verbi modali. Secondo l'analisi quantitativa dei costrutti infinitivi con i verbi modali in italiano moderno, eseguita da Skytte (1983), con sapere e volere ci sia una maggiore tendenza alla posposizione del pronome atono che con dovere e potere.

Per poter accettare queste ipotesi, sarebbe opportuno effettuare un'attenta analisi statistica e un confronto più approfondito delle due prospettive: quella sincronica e quella diacronica.

A prescindere dalle diverse manifestazioni linguistiche dei costrutti modali nella struttura superficiale, la categoria della modalità esprime la funzione espressiva e pragmatica del linguaggio e pertanto dovrebbe essere considerata universale. La modalità secondo Simone (1995: 236) rientra tra quelle categorie che offrono "una base essenziale per la ricerca di universali linguistici”.

Per i motivi già menzionati, la posizione dei pronomi atoni, nella nostra analisi, non sarà considerata un fattore decisivo che può incidere sulla struttura profonda della frase né sulla funzione sintattica dell'infinito nelle costruzioni rette dai verbi modali.

\subsection{La scelta dell'ausiliare}

L'ausiliare del verbo modale nell'uso assoluto è sempre avere (ho potuto / dovuto / voluto). Come in genere in italiano moderno, se l'ausiliare del verbo al posto dell'infinito è avere, anche il costrutto modale presenterà avere:

[...] li letterati fuori di lingua Italica non avrebbono potuto avere questo servigio... (Convivio, I, IX, 273)

E nel vero, se io potuto avessi onestamente per altra parte menarvi a quello che io desidero... (Decameron, I, 9) 
Ma per alcun caso avrebbeti l'ira potuto inducere a fare alcuno omicidio o a dire villania a persona o a fare alcuna altra ingiuria? (Decameron, I, 1, 34)

Fanno eccezione a questa regola le costruzioni con i verbi riflessivi al posto dell'infinito, nelle quali i pronomi atoni si cliticizzano al verbo reggente:

[...] di chi si fussi potuto servire... (Principe, VII, 15)

[...] ma vi si fussono potuti distendere... (Dell'arte della guerra, I, 289)

[...] se io dalla verità del fatto mi fossi scostare voluta... (Decameron, IX, 5, 620)

Se l'ausiliare del verbo lessicale è essere, in italiano antico, il costrutto ristrutturato può presentare sia essere (unità), che avere (dualità) ${ }^{19}$. Nei costrutti modali con i verbi intransitivi, precisa Telve (2007), il toscano duetrecentesco privilegiava l'ausiliare essere, mentre il costrutto con avere si diffonde nella scrittura letteraria all'epoca umanistica e rinascimentale. La grammatica di Pietro Bembo (1966: 154), ammette per i costrutti di questo tipo solo il verbo essere:

"Là dove se si dicesse se io havessi voluto andare dietro a' sogni, non si potrebbe poscia sciogliere et dire: se io havessi andato dietro a'sogni; percioché queste voci così dette non tengono. Fassi questo medesimo co' verbi voluto et potuto, che si dice: son voluto venire, son potuto andare, percioché son venuto et sono andato si scioglie; là dove ho venuto et ho andato non si scioglie".

Se l'infinito è un verbo intransitivo, l'ausiliare è essere:

Il Saladino conobbe costui ottimamente essere saputo uscire del laccio... (Decameron, I, 3, 47)

[...] e quale uscio ti fu mai in casa tua tenuto, quando tu colà dove io fossi, se' voluto venire? (Decameron, VII, 5, 476)

Ghismunda, parendomi conoscere la tua vertù e la tua onestà, mai non mi sarebbe potuto cader nell'animo... (Decameron, 4,1, 278)

Fanno eccezione però i costrutti modali con i verbi di moto usati in un senso traslato:

[...] chi vince non vuole amici sospetti e che non lo aiutino nelle avversità, chi perde, non ti riceve, per non avere tu voluto con le arme in mano correre la fortuna sua. (Principe, XXI, 44)

Nell'esempio citato si tratta dell'uso fraseologico del verbo correre. Il verbo correre regge un complemento oggetto (la fortuna sua) ed è usato transitivamente.

${ }^{19}$ Cfr. Egerland (2010: 830). 
La scelta tra essere e avere con i verbi che in uso isolato richiedono essere, sottolinea Skytte (1983), può dipendere dalla distinzione semantica collegata alla distinzione unità-dualità. Se l'ausiliare del costrutto è essere, viene messa in rilievo l'azione stessa espressa dall'infinito, modificata con il verbo modale, mentre l'ausiliare avere esalta il carattere modale della costruzione. Similmente, la scelta del verbo avere nelle costruzioni con infiniti intransitivi viene interpretata da Nencioni (1989: 294) come la tendenza "a rendere il verbo modale autonomo dal verbo modalizzato con l'applicargli l'ausiliare suo proprio".

L'applicabilità di queste ipotesi sarà esaminata nei costrutti col verbo essere al posto dell'infinito e nei costrutti con si passivante, perché l'italiano antico nei costrutti del genere ammette entrambi gli ausiliari.

Quando l'infinito è il verbo essere, i due ausiliari alternano ${ }^{20}$.

[...] benché io sommamente disiderassi, se essere fosse potuto, di fare... (Elegia di Madonna Fiammetta, V, 43)

Pòrtalti in pace, ché quello che stanotte non è potuto essere, sarà un'altra volta... (Decameron, VIII, 7, 550)

Quegli che là entro rimasono, in parte dalle ragioni di Tito al parentado e alla sua amistà indotti, ed in parte spaventati dall'ultime sue parole, di pari concordia diliberarono essere il migliore d'aver Tito per parente, poi che Gisippo non aveva esser voluto, che aver Gisippo per parente perduto e Tito nimico per acquistato. (Decameron, X, 8, 702)

Con il verbo essere al posto dell'infinito, trattandosi di un verbo di stato, è messo in rilievo il carattere modale della situazione, e le distinzioni semantiche osservate da Skytte (1983) riguardo alla messa in rilievo dell'azione stessa (essere), oppure della modalità (avere), non sono riscontrate nelle costruzioni del genere.

Similmente, nelle costruzioni ristrutturate con il si passivante in italiano antico compaiono entrambi gli ausiliari:

[...] che tutti i denti si sarebbero loro potuti trarre... (Decameron, VI, 410)

Il che veggendo la gente, sì gran romore in lode di santo Arrigo facevano che $\mathrm{i}$ tuoni non si sarieno potuti udire. (Decameron, II, 1, 80)

[...] sanza ciò le grandissime cose non s'arebbono potute mettere in compimento... (Brunetto Latini, Rettorica, 26) ${ }^{21}$

${ }^{20}$ L'italiano moderno avrebbe solo avere in questi casi (Egerland 2010; Telve 2007). Sebbene poco frequente, secondo Skytte (1983: 99) sarebbe possibile anche essere.

${ }^{21}$ Cfr. Egerland (2010: 833). 
Come risulta da questi esempi, la scelta dell'ausiliare in italiano antico non è determinata dalla posizione del pronome atono. Anche se accettassimo l'ipotesi che la posizione del pronome atono e la scelta dell'ausiliare incidano sull'unità del costrutto modale, non potremmo con precisione identificare il tipo del predicato nell'ultimo esempio citato in base ai criteri puramente sintattici. Mentre lo spostamento del si passivante, secondo l'ipotesi presentata da Skytte (1983), potrebbe essere indizio del costrutto di unità, l'uso dell'ausiliare avere nel costrutto modale con si passivante indica il carattere bifrasale del costrutto.

Per quanto riguarda la scelta dell'ausiliare, nel corpus analizzato, non sono notati importanti scostamenti dalla norma dell'italiano standard, tranne quelli individuati nel presente capitolo.

\subsection{L'infinito composto}

L'infinito composto è una forma autonoma dal punto di vista temporale. Lo stesso si può dire dell'aspetto verbale: l'infinito composto è una forma assolutamente univoca dal punto di vista aspettuale e "implica sempre il valore aspettuale di compiutezza" (Bertinetto 2001: 11).

L'infinito composto può essere introdotto da tutti i verbi modali, con l'eccezione del verbo sapere, per i suoi tratti semantici specifici ${ }^{22}$ :

E pensando seco stesso che questa potrebbe essere tal femina o figliuola di tale uomo, che egli non le vorrebbe aver fatta quella vergogna d'averla a tutti i monaci fatta vedere, s'avvisò di... (Decameron, I, 4, 49) (1'infinito passato)

[...] la quale a lei potrebbe essere stata contraria. (Convivio, III, IX, 308) (l'infinito passato)

[...] comprendendo per le parole di Nuto che a lui dovrebbe poter venir fatto ${ }^{23} \mathrm{di}$ quello che egli disiderava. (Decameron, III, 1, 188) (l'infinito presente passivo)

Il carattere bifrasale è ovvio nei costrutti con il verbo volere che dimostra maggiore autonomia sintattica rispetto agli altri verbi modali, dato che può reggere una completiva oggettiva esplicita introdotta da che, oppure un complemento oggetto nominale ${ }^{24}$.

Con il verbo volere sono tra l'altro frequenti spostamenti semantici che dipendono dall'incompatibilità logica tra il verbo volere che si riferisce all'azione futura, e il verbo nell'infinito che esprime azione passata (Skytte, 1983: 90):

${ }^{22}$ Il verbo sapere può reggere un'oggettiva implicita all'infinito introdotta dalla preposizione $d i$, ma in questo caso non ha più il valore modale.

${ }^{23}$ Si noti l'uso pleonastico del verbo dovere.

${ }^{24}$ Cfr. Skytte (1983: 83); Skytte \& Salvi (1991: 462). 
[...] io vorrei piuttosto essere stato morto che vivo, veggendo i giovani andar dietro alle vanità ed udendogli giurare e spergiurare, andare alle taverne, non visitar le chiese e seguir più tosto le vie del mondo che quella di Dio. (Decameron, I, 1, 34)

La semantica di volere con l'infinito presente passivo è piuttosto pretendere, desiderare:

[...] e altri, che senza dire voglion essere serviti e intesi; e altri che non vogliono che '1 servo si muova a fare quello che' è mestieri, se nol comandano. (Convivio, I, VI, 270)

Questo signore, cioè queste Canzoni [...] comandano e vogliono essere sposte a tutti coloro alli quali può venire sì lo loro intelletto... (Convivio, I, VII, 271)

L'uso dell'infinito composto retto dai verbi dovere o potere, è tipico del senso epistemico:

E perocché potrebbe alcuno avere domandato dove questo mirabile piacere appare in costei... (Convivio, III, VIII, 307) (l'infinito passato)

Qualunque affari, qualunque altre cagioni costà trovasti, già deono essere finite... (Elegia di Madonna Fiammetta, V, 37) (l'infinito presente passivo)

Inteso può essere sufficientemente, per le prenarrate parole... (Convivio, II, VIII, 285) (l'infinito presente passivo)

La modalità epistemica viene intesa come l'espressione dell'opinione del parlante circa la verità dell'enunciato ${ }^{25}$. Una spiccata componente espressiva che riguarda l'atteggiamento del parlante nell'atto illocutorio, contribuisce alla coerenza sintattica e semantica dei costrutti modali con significati epistemici.

L'unità dei costrutti modali con l'infinito presente passivo si rispecchia anche nella possibilità di trasmettere la struttura passiva al verbo reggente:

Questi Lombardi cani, li quali a chiesa non sono voluti ricevere, non ci si voglion più sostenere! (Decameron, I, 1, 32)

Oh quante volte, se i solleciti amanti avessero saputo questo, forse sarei stata potuta ingannare, se alcuno malizioso sé Panfilo avesse finto a cotali punti! (Elegia di Madonna Fiammetta, V, 38)

Quest'uso è assai raro nell'italiano moderno ${ }^{26}$.

${ }^{25}$ Cfr. Halliday \& Matthiessen (2004); Lyons (1977).

${ }^{26}$ Cfr. Skytte (1983); Telve (2007). 


\subsection{La posizione della negazione}

L'infinito negato indipendentemente viene considerato uno dei fattori sintattici che possano creare dualità ed incidere sulla coerenza del costrutto (Skytte 1983). Se la negazione riguarda l'intero predicato complesso, contribuisce all'unità sintattica del costrutto verbo modale + infinito:

[...] li letterati fuori di lingua Italica non avrebbono potuto avere questo servigio. (Convivio, I, IX, 273)

A che fine ami tu questa tua donna, poi che tu non puoi sostenere la sua presenza? (Vita nuova, XVIII, 244)

Nel corpus si ritrovano rari esempi di costruzioni modali nelle quali è negato soltanto il verbo all'infinito:

Debbe, adunque, uno principe non avere altro obietto né altro pensiero, né prendere cosa alcuna per sua arte, fuora della guerra e ordini e disciplina di essa... (Principe, XIII, 29)

[...] debbe, s'egli è prudente, non si curare ${ }^{27}$ del nome del misero... (Principe, XV, 31)

L'infinito negato indipendentemente dal verbo modale negli esempi citati conferisce all'enunciato un valore deontico attenuato: la necessità viene espressa come un consiglio, un suggerimento e non come un divieto (debbe uno principe non avere: non debbe avere; debbe non si curare: non debbe curarsi). Il valore deontico attenuato invece non comporta alcuna modifica sostanziale nella coerenza sintattico-semantica del costrutto che è considerato monofrasale.

\subsection{Il valore aspettuale di verbo modale + infinito}

Il valore aspettuale dell'infinito potrebbe incidere sull'unità del costrutto verbo modale + infinito ${ }^{28}$.

Secondo Skytte (1983), i verbi modali sono considerati "neutri" dal punto di vista aspettuale, e formano un'unità verbale con l'infinito, che assume la dominanza aspettuale:

[...] ne la quarta dico che volendo dire d'Amore, non so da qual parte pigli matera, e se la voglio pigliare da tutti, convene che io chiami la mia inimica, madonna la Pietade... (Vita nuova, XIII, 241)

Nell'esempio citato troviamo due costruzioni modali con il verbo volere, con diversi valori aspettuali, definiti dal verbo all'infinito. Mentre

\footnotetext{
${ }^{27}$ Si noti l'insolita interposizione del «si» riflessivo.

${ }^{28}$ Cfr. Skydsgaard (1977); Skytte (1983).
} 
la prima costruzione potrebbe essere considerata imperfettiva (l'aspetto è tra l'altro definito dalla forma verbale del verbo modale: volendo), il verbo pigliare assume la dominanza aspettuale nella seconda costruzione che è considerata perfettiva (voglio pigliare).

L'uso dei verbi essere e avere al posto dell'infinito secondo Skytte (1983: 81), sarebbero "un fattore che normalmente crea la dualità", essendo "neutri" dal punto di vista aspettuale, appunto come il verbo modale. Esaminando il valore aspettuale di verbo modale + infinito, Skydsgaard (1977) considera il verbo modale come imperfettivo e dominante in senso aspettuale. Cambiando il significato, i verbi essere e avere, nell'unione con il verbo modale, possono creare un'unità sintattica, dell'aspetto verbale ben definito (ibid.):

[...] l'anima umana esser vuole naturalmente con tutto desiderio. (Convivio, III, II, 298)

Ov'è da sapere che la divina bontà in tutte le cose discende; e altrimenti essere non potrebbono... (Convivio, III, VII, 305)

Il verbo essere negli esempi citati assume il significato del verbo esistere e diventa parte di un'unità verbale imperfettiva.

Nei seguenti esempi il verbo essere è semanticamente affine ai verbi succedere, accadere e il valore aspettuale delle costruzioni infinitive potrebbe essere considerato perfettivo:

[...] altrimenti non si continuerebbe la umana spezie da ogni parte, che essere non può. (Convivio, III, VII, 305)

E ciò non potea né dovea essere se non per ispeziale fine da Dio inteso... (Convivio, $\mathrm{IV}, \mathrm{V}, 324)$

Similmente, il verbo avere assumendo il significato di cogliere definisce l'aspetto della costruzione modale:

[...] valendoti della negligenza loro, puoi avere facilmente occasione (= puoi cogliere l'occasione) di uscire loro delle mani. (Dell'arte della guerra, VI, 349)

Se ne può concludere che i verbi essere e avere, cambiando i significati di base, possono definire l'aspetto della costruzione modale creando un'unità verbale con il verbo modale. Al posto del termine "neutro", usato da Skytte (1983), sarebbe più opportuno usare il termine "dominante" dal punto di vista aspettuale. Le marche dell'aspetto nella lingua italiana sono raramente incluse nel tema o nelle desinenze e il contesto gioca un ruolo cruciale nell'interpretazione dell'aspetto verbale.

L'infinito potrebbe essere considerato "neutro" dal punto di vista aspettuale nelle quando fa parte del predicato nominale, con il verbo essere usato come copula: 
Dolcissima Morte, vieni a me, e non m'essere villana, però che tu dèi essere gentile, in tal parte se' stata! (Vita nuova, XXIII, 249)

[...] perché li savi dicono che la faccia del dono dee esser simigliante a quella del ricevente... (Convivio, I, VIII, 272)

Primamente, perché la virtù dee essere lieta, e non trista in alcuna sua operazione.

(Convivio, I, VIII, 272)

Nelle costruzioni con il predicato nominale, i verbi modali conferiscono al predicato nominale l'espressione della modalità, assolvendo la funzione di servizio nei confronti del verbo all'infinito. Queste costruzioni sono considerate monofrasali dell'aspetto verbale imperfettivo. La possibilità di omettere la parte nominale del predicato sottolinea la coerenza sintattica e semantica:

[...] conciossiacosaché l'uno contrario non sia fattore dell'altro, né possa essere per la prenarrata cagione. (Convivio, IV, X, 332)

\section{CONSIDERAZIONI CONCLUSIVE}

In questo nostro contributo abbiamo analizzato la struttura dei costrutti infinitivi introdotti dai verbi modali, esaminando e confrontando le loro proprietà semantiche e sintattiche come tratti distintivi che potrebbero incidere sull'unità del sintagma verbale e differenziare la costruzione monofrasale da quella bifrasale.

L'analisi approfondita dei valori semantici delle costruzioni modali e della categoria della modalità in genere, conferma che l'accentuata soggettività del parlante e il forte legame che si crea tra il parlante e il suo enunciato contribuiscono alla coerenza semantica del costrutto.

Esaminando l'effetto dei vari tratti sintattici sull'unità della costruzione modale (la posizione dei pronomi atoni, la posizione della negazione, l'uso dell'infinito composto, la scelta dell'ausiliare) siamo pervenuti alla conclusione che le modifiche nella struttura superficiale della frase hanno un ruolo secondario rispetto alla coerenza semantico-pragmatica.

Come risulta dall'analisi delle costruzioni ristrutturate individuate nel corpus, la scelta dell' ausiliare non è determinata dalla posizione del pronome atono e non incide sull'unità del costrutto. La posizione dei pronomi atoni non comporta nessuna modifica sul piano semantico né può incidere sulla funzione sintattica dell'infinito.

Diversi valori aspettuali del costrutto verbo modale + essere / avere, definiti dal verbo all'infinito, che cambiando il significato di base assume la dominanza aspettuale, confermano che i verbi modali assolvono la funzione di servizio nei confronti del verbo all'infinito. 
I tratti sintattici che si discostano dall'italiano moderno, fra i quali la possibilità di trasmettere la struttura passiva al verbo modale, notata nei rari esempi delle costruzioni modali passive nelle opere di Boccaccio e sporadiche oscillazioni nella scelta dell'ausiliare del verbo modale, rispecchiano la coerenza sintattico-semantica e confermano l'ipotesi sull'unità del predicato.

L'unico costrutto di tipo bifrasale individuato nel corpus è formato dall'infinito composto (sia di valore temporale che di valore passivo) introdotto dal verbo volere, che tra l'altro dimostra la maggiore autonomia sintattica tra $\mathrm{i}$ verbi modali. La possibilità di sostituire un costrutto infinitivo introdotto dal verbo volere con una proposizione oggettiva esplicita, insieme agli spostamenti semantici di questo verbo, il quale nel costrutto con l'infinito viene spesso usato nell'accezione di desiderare, pretendere, rispecchia il carattere bifrasale del costrutto.

Nel determinare il tipo di costrutto, vanno presi in considerazione $\mathrm{i}$ fattori semantici come un forte elemento coesivo che dovrebbe essere dominante rispetto ai fattori sintattici percepibili nella struttura superficiale della frase. Altrimenti, tenendo presenti diverse rappresentazioni sintattiche delle costruzioni modali in italiano antico, dovremmo ipotizzare una diversa interpretazione delle costruzioni esaminate e della stessa categoria di modalità durante il percorso evolutivo della lingua italiana.

\section{BIBLIOGRAFIA}

Ageno, F. B. (1978). Verbo: Infinito. In U. Bosco (a cura di), Enciclopedia Dantesca. Appendice (pp. 268-292). Roma: Istituto della Enciclopedia italiana fondata da Giovanni Treccani.

Bembo, P. (1966). Prose e rime, a cura di C. Dionisotti. Torino: Utet.

Bertinetto, M. (2001). Sulle proprietà tempo-aspettuali dell'infinito in italiano, versione preliminare destinata agli atti del $35^{\circ}$ Congresso Internazionale della Società di linguistica Italiana, 9-5. (Testo disponibile al sito: http://linguistica.sns.it/QLL/QLL01/PMB.Infinito.pdf).

Bybee J., Perkins R. \& Pagliuca W. (1994). The Evolution of Grammar.

Chicago: The University of Chicago Press.

Dardano, M. (1963). Sintassi dell'infinito nei "Libri della Famiglia" di L.

B. Alberti. Annali della Scuola Normale di Pisa, XXIII, 83-135.

Dardano, M. \& Trifone, P. (1997). La nuova grammatica della lingua italiana. Bologna: Zanichelli Editore.

Egerland, V. (2010). Frasi subordinate all'infinito. In G. Salvi \& L. Renzi (a cura di), Grammatica dell'italiano antico (Vol. 2, pp. 817-881). Bologna: Il Mulino.

Fornaciari, R. (1881). Sintassi italiana dell'uso moderno. Firenze: Sansoni. 
Halliday, M. A. K. (1970). Functional Diversity in Language as Seen from a Consideration of Modality and Mood in English. Foundations of Language, 6, 322-361.

Halliday, M. A. K. \& Matthiessen, M. I. M. (2004). An introduction to functional grammar. New York: St Martin's Press.

Lo Cascio, V. (1970). Strutture pronominali e verbali italiane. Bologna: Zanichelli.

Lyons J. (1977). Semantics. Cambridge: Cambridge University Press.

Nencioni, G. (1989). Saggi di lingua antica e moderna. Torino: Rosenberg \& Sellier.

Palmer, F. R. (1986). Mood and Modality. Cambridge: Cambridge University Press.

Palmer, F. R. (1990). Modality and the English Modals. London: Longman. Palmer, F. R. (2003). Modality in English: Theoretical, Descriptive and Typological issues. In R. Facchinetti, M. Krug \& F. R. Palmer (a cura di), Modality in Contemporary English (pp. 1-17). Berlin / New York: Mouton de Gruyter.

Pietrandrea, P. (2005). Epistemic Modality: Functional Properties and the Italian System. Amsterdam / Philadelphia: John Benjamins Publishing Company.

Rizzi, L. (1976). Ristrutturazione. Rivista di Grammatica Generativa, 1, $1-54$.

Rohlfs, G. (1969). Grammatica storica della lingua italiana e dei suoi dialetti. Sintassi e formazione delle parole. Torino: Giulio Einaudi editore.

Schmitt Jensen, J. (1973). L'infinitif et la construction relative en français et en italien contemporains. Revue Romane, VIII, 122-132.

Serianni, L. (1989). Grammatica italiana. Italiano comune e lingua letteraria, con la collaborazione di A. Castelvecchi. Torino: UTET.

Simone R. (1995). The Language User in Saussure (and after). In L. Formigari \& D. Gambarara (a cura di), Historical Roots of Linguistic Theories (pp. 233-249). Amsterdam: John Benjamins.

Simone, R. \& Amacker, R. (1977). Verbi modali in italiano. Italian linguistics, 3, 7-12.

Skydsgaard, S. (1977). La combinatoria sintáctica del infinitivo español. Madrid: Castalia.

Skytte, G. (1983). La sintassi dell'infinito in italiano moderno. Denmark: Etudes Romanes de l'Université de Copenhague.

Skytte, G. \& Salvi, G. (1991). Frasi subordinate all'infinito. In G. Salvi \& L. Renzi (a cura di), Grammatica italiana di consultazione, (Vol. 2, pp. 483-571). Bologna: Il Mulino. 
Telve, S. (2007). Essere o avere? Sull'alternanza degli ausiliari coi modali potuto, voluto (e dovuto) davanti a infiniti inaccusativi in italiano antico e moderno. In V. Della Valle \& P. Trifone (a cura di), Studi linguistici per Luca Serianni (pp. 313-325). Roma: Salerno Editrice.

Wurmbrand, S. (1994). Infinitives (tesi di dottorato di ricerca non pubblicata). Testo disponibile al sito: www.ai.mit.edu/projects/dm/theses/wurmbrand98.pdf.

\section{THE UNITY OF THE PREDICATE IN MODAL INFINITIVE CONSTRUCTIONS}

\section{Summary}

The subject of this scientific paper is examining syntactic functions of infinitive in modal infinitive constructions, focusing in particular on the unity of the predicate. Modal infinitive constructions are analyzed by examining their semantic values as well as following syntactic features that might influence the unity of the predicate and make a distinction between mono-phrasal and bi-phrasal construction: the position of unstressed pronouns, the choice of auxiliary, the use of perfect and passive infinitives, the position of negation, the aspect. The corpus consists of the literary works of the authors who marked the development of Italian prose discourse. The paper is based on qualitative research methodology without reporting detailed statistics. Our objective is providing a systematic syntactic overview of modal infinitive constructions in older Italian language, as well as offering a more detailed examination of particular aspects of infinitive structures, such as the unity of the predicate, which has been largely neglected in Italian grammar books.

Keywords: infinitive, modal verbs, mono-phrasal construction, bi-phrasal construction, unity of the predicate, older Italian language. 